\title{
Conservation of Properties of Outer Membranes Protein Across Host Genera of Pasteurella multocida Suggests Common Mechanism of Action
}

\author{
Neetesh Pandey, Monendra Grover* and Anil Rai
}

Centre for Agricultural Bio-Informatics, ICAR-Indian Agricultural Statistics Research Institute, Library Avenue, PUSA, New Delhi, India

\begin{abstract}
Pasteurella multocida is a non-motile coccobacillus pathogenic Gram-negative bacterium and belongs to Pasteurellaceae family. Pasteurella multocida causes diseases in economically important animals and birds in developing and developed countries. Haemorrhagic septicaemia in cattle and buffaloes and other diseases like fowl cholera (turkey, chicken, and duck), Septicaemic pasteurellosis (sheep, pig, and goat) and Snuffles (rabbit) are caused by Pasteurella multocida. In this analysis we have taken three outer membrane proteins (vacJ, ompW and skp) from $P$. multocida which are involved in infectious diseases of animals and birds. The literature shows that all three outer membrane proteins are infectious in nature. This is supported by multiple sequence alignment and analysis of physicochemical properties of protein sequences encoded by vacJ, ompW and skp outer membrane protein families. The studied proteins are similar with respect to number of positively and negatively charged amino acids, molecular weight, Theoretical pl, instability index and grand average of hydropathicity (GRAVY) in the proteins from $P$. multocida strains infecting different genera. The domains, transmembrane helices, twin arginine signal peptides and $\boldsymbol{B}$-barrels are also broadly similar. This suggests common mechanism of action of these proteins across host genera.
\end{abstract}

Keywords: Pasteurella multocida; vacJ; ompW; skp

Abbreviations: ompW: Outer Membrane Protein W; HS: Haemorrhagic Septicaemia; GRAVY: Grand Average of Hydropathicity

\section{Introduction}

Pasteurella multocida has been classified on the basis of properties and reactions of blood serum and disease that are designated by five capsular serogroups A, B, C, D, E, F, and 16 types of serotypes [1]. The main hosts of haemorrhagic septicaemia infection and disease are buffalo and cattle but other animals like goats, pigs, deer, sheep and camel are also susceptible to it. The disease caused by Pasteurella multocida involves an aerosol transmission of pathogens through the air by means of inhalation of infectious particles. The respiratory tracts of warm-blooded animals may be affected by diseases like haemorrhagic septicaemia, fowl cholera and snuffles [2]. Pasteurella multocida serotype B: 2 is the etiological agent of haemorrhagic septicaemia (HS) in cattle and buffaloes, a disease that causes significant economic losses to the dairy industry in India [3]. Haemorrhagic septicaemia is caused by two particular serotypes of Pasteurella multocida: B: 2 and E: 2 . The serotype B: 2 is the Asian serotype and E: 2 is the African serotype. Haemorrhagic septicaemia has an extensive distribution world-wide especially in Africa and South East Asia. It is known to be associated with the localisation of bacteria in the tonsils of living buffalo, showing that animals can become transporters [4].

The lipopolysaccharide and carbohydrate surface molecules including a range of virulence factors are expressed by Pasteurella multocida. The capsule of polysaccharide belonging to serogroups $\mathrm{A}$ and B has been shown in strains of Pasteurella multocida to help decline phagocytosis by cell of host immune .Type A capsule has also been shown to help decline complement mediated lysis $[5,6]$. Outer membrane proteins play an important role in pathological process of Pasteurellosis. These are broadly distributed in Gram-negative bacteria which are involved in various mechanisms of pathogenesis.

Many pathogenic bacterial strains belonging to the Pasteurellaceae family have numerous surface exposed virulence elements including vacJ-like lipoproteins. vacJ proteins are neither involved in the invasion of epithelial cells nor in intracellular movement, but are required for intercellular spread. The vacj like lipoprotein in case of mutant is capable of forming bacterium-containing membranous protrusions within the infected cell by Pasteurella multocida bacterial strains. Sequencing and cloning of vacj lipoprotein region indicated that the gene-vacj encoded a $28.0 \mathrm{kDa}$ protein owning a signal peptide at the $\mathrm{N}$-terminus, which contained the motif characteristic of lipoproteins [7].

skp gene is involved in the biogenesis of OMPs and histone like proteins that bind to DNA that is transported by skp [8]. Skp plays an important role for surface presentation and folding of the $\alpha$-domain of the auto transporter [9]. The gene ompW is present in the bacterial outer membrane grown at $37^{\circ} \mathrm{C}$. It abruptly disappears at $23^{\circ} \mathrm{C}$ with the parallel acquisition of colicin $S 4$ resistance by the cells [10].

As mentioned before the P. multocida strains affect different animals and birds. In this study we attempted to find out whether the proteins encoded by the genes vacJ, skp, ompW have similar mechanism of action in different host genera. To our surprise we found that the mechanism of action of proteins encoded by these genes is possibly broadly similar across divergent host genera. This may be useful in development of a common vaccine against strains of $P$. multocida infecting different host genera.

\section{Materials and Methods}

The sequences of outer membrane protein (vacJ, ompW, skp) were retrieved from NCBI nucleotide database (http://www.ncbi.nlm.nih. gov). These sequences were from strains of $P$. multocida affecting cattle, buffalo, duck, pig, sheep, quail, rabbit, chicken and turkey. These strains are involved in different diseases like Haemorrhagic septicaemia, Fowl cholera, Septicaemia pasteurellosis, Snuffles etc.

The multiple sequence alignment was performed by clustal omega

*Corresponding author: Monendra Grover, Centre for Agricultural BioInformatics, ICAR-Indian Agricultural Statistics Research Institute, Library Avenue, PUSA, New Delhi, India, E-mail: monendra_grover@yahoo.com

Received April 07, 2016; Accepted April 18, 2016; Published April 25, 2016

Citation: Pandey N, Grover M, Rai A (2016) Conservation of Properties of Outer Membranes Protein Across Host Genera of Pasteurella multocida Suggests Common Mechanism of Action. Mol Biol 5: 162. doi:10.4172/2168-9547.1000162

Copyright: @ 2016 Pandey N, et al. This is an open-access article distributed under the terms of the Creative Commons Attribution License, which permits unrestricted use, distribution, and reproduction in any medium, provided the original author and source are credited. 
(http://www.ebi.ac.uk/Tools/msa/clustalo/) to compare skp, vacJ and ompW sequences from different host genera.

The domain analysis has been done with the help of local sequence alignment. The local alignment was performed using BLAST (http:// blast.ncbi.nlm.nih.gov/Blast.cgi) against non-redundant protein sequences database ( $\mathrm{nr}$ database) for searching the conserved domains for all 23 sequences that belong to vacJ, skp and ompW outer membrane proteins. The domain search analysis was completed with the conserved Domain Architecture Retrieval Tool Program (http://www.ncbi.nlm. nih.gov/Structure/cdd/).

The 3D structure and biological activity of proteins depend on the physicochemical properties. The computed parameters in this study include their constituent amino acids, negatively charged amino acids, extinction coefficient, theoretical pI, amino acid composition, grand average of hydropathicity (GRAVY) and aliphatic index [11]. The calculation of physcio-chemical properties was done using ProtParam tool. (http://web.expasy.org/protparam/).

Transmembrane helices refer to membrane proteins where the transmembrane regions are embedded in a phospholipid bilayer and the extra membrane domains are surrounded by water. This waterexposed polypeptide can adopt a diverse array of folds, whereas the physical and chemical constraints imposed by the lipid bilayer appear to restrict the structural diversity of the embedded protein domain (Neuberger, et al., 2003). Prediction of transmembrane helices was done through TMHMM Server v. 2.0 (http://www.cbs.dtu.dk/services/ TMHMM-2.0/).

Twin-arginine signal peptides are carried by proteins and exported to periplasmic compartment or sec-dependent translocation pathway. The prediction of bacterial Tat signal peptides was done using TatP 1.0 Server (http://www.cbs.dtu.dk/services/TatP/) [12].

In this analysis the BOMP tool (http://services.cbu.uib.no/tools/ bomp) was used for prediction of transmembrane B-barrel outer membrane proteins, which is capable of predicting the transmembrane strands and the topology of $\beta$-barrel outer membrane proteins of Gram-negative bacteria.

\section{Result}

\section{Multiple sequence alignment}

The sequences of outer membrane protein like vacJ, ompW, skp were retrieved from NCBI nucleotide database and analysed using MSA tool. The retrieved sequences were from Indian strains of Pasteurella multocida infecting cattle, buffalo, duck, pig, sheep, quail, rabbit, chicken and turkey. The analysis of vacJ outer membrane protein revealed that, the alignment score of the sequences was between 98.37 to 100 percent and proline, aspartic acid, glutamic acid, serine and threonine are the variable amino acids. The similar analysis with ompW reveals that the alignment score of these sequences is between 96.08 to 100 percent and valine, glycine, aspartic acid, aspargine, tyrosine and lysine were variable amino acids. The protein sequences of skp outer membrane proteins were 100 percent similar. Thus it can be inferred that the mechanism of action of skp protein is identical in all the Pasteurella multocida strains affecting different genera.

\section{Conserved domain prediction}

The protein domains often form functional units. Keeping this in view, conserved domain analysis was performed using CDD tool to tease out any possible similarities/differences in mechanism of action of skp, ompW and vacJ proteins across the host genera. Four conserved domains were predicted (Table 1). Domain COG2853, pfam0433 and PRK15091 are present in all 23 proteins and domain smart0093 was present only in protein sequences that were encoded by skp gene. The process of biogenesis is initiated by COG2853 domain that is present in the outer membrane region. This domain is present in the Outer Membrane of bacteria grown at $37^{\circ} \mathrm{C}$, but it totally vanishes at $23^{\circ} \mathrm{C}$. The $\mathrm{E}$ value of domain pfam04333 is smaller in comparison to other

\begin{tabular}{|c|c|c|c|c|c|c|}
\hline Protein_id & Protein Name & Animal name & COG2853 & pfam 04333 & PRK15091 & smart00935 \\
\hline AFQ32090.1 & vacJ outer membrane lipoprotein & Cattle & $5.66 \mathrm{E}-118$ & $1.81 \mathrm{E}-106$ & $3.72 \mathrm{E}-105$ & Not present \\
\hline AFQ32091.1 & vacJ outer membrane lipoprotein & Duck & $2.94 \mathrm{E}-118$ & 1.93E-106 & $5.56 \mathrm{E}-105$ & Not present \\
\hline AFQ32092.1 & vacJ outer membrane lipoprotein & Chicken & $2.94 \mathrm{E}-118$ & 1.93E-106 & $5.56 \mathrm{E}-105$ & Not present \\
\hline AHW46101.1 & vacJ outer membrane lipoprotein & Quail & $5.66 \mathrm{E}-118$ & $1.81 \mathrm{E}-106$ & $3.72 \mathrm{E}-105$ & Not present \\
\hline AHW46102.1 & vacJ outer membrane lipoprotein & Pig & $2.94 \mathrm{E}-118$ & 1.93E-106 & $5.56 \mathrm{E}-105$ & Not present \\
\hline AHW46103.1 & vacJ outer membrane lipoprotein & Sheep & $1.11 \mathrm{E}-116$ & $1.42 \mathrm{E}-105$ & 8.06E-107 & Not present \\
\hline AHW46104.1 & vacJ outer membrane lipoprotein & Buffalo & $5.66 \mathrm{E}-118$ & $1.81 \mathrm{E}-106$ & $3.72 \mathrm{E}-105$ & Not present \\
\hline AHW46105.1 & vacJ outer membrane lipoprotein & Goat & 1.11E-116 & $1.42 \mathrm{E}-105$ & 8.06E-107 & Not present \\
\hline AHW46106.1 & vacJ outer membrane lipoprotein & Rabbit & 7.11E-118 & 4.15E-106 & $3.54 \mathrm{E}-106$ & Not present \\
\hline AHX71787.1 & Outer membrane protein $\mathrm{W}$ & Sheep & $1.54 \mathrm{E}-85$ & $9.06 \mathrm{E}-81$ & $3.58 \mathrm{E}-89$ & Not present \\
\hline AHX71788.1 & outer membrane protein $\mathrm{W}$ & Buffalo & 3.03E-85 & $7.62 E-81$ & 4.03E-89 & Not present \\
\hline AHX71789.1 & outer membrane protein $\mathrm{W}$ & Goat & 3.03E-85 & 4.03E-89 & 4.03E-89 & Not present \\
\hline $\mathrm{AHX71790.1}$ & outer membrane protein $\mathrm{W}$ & Duck & $1.04 \mathrm{E}-83$ & $6.42 \mathrm{E}-79$ & $1.20 \mathrm{E}-87$ & Not present \\
\hline $\mathrm{AHX71791.1}$ & outer membrane protein $\mathrm{W}$ & Chicken & $5.26 \mathrm{E}-86$ & $3.10 \mathrm{E}-90$ & $3.10 \mathrm{E}-90$ & Not present \\
\hline $\mathrm{AHX71792.1}$ & outer membrane protein $\mathrm{W}$ & Turkey & $5.46 \mathrm{E}-83$ & 1.13E-77 & $1.10 \mathrm{E}-86$ & Not present \\
\hline AHX71793.1 & outer membrane protein $\mathrm{W}$ & Rabbit & $1.54 \mathrm{E}-85$ & $3.58 \mathrm{E}-89$ & $3.58 \mathrm{E}-89$ & Not present \\
\hline AHX71780.1 & skp outer membrane protein & Sheep & $6.58 \mathrm{E}-30$ & $4.25 \mathrm{E}-19$ & $1.80 \mathrm{E}-18$ & 3.14E-22 \\
\hline $\mathrm{AHX71781.1}$ & skp outer membrane protein & Buffalo & $6.58 \mathrm{E}-30$ & $4.25 \mathrm{E}-19$ & $1.80 \mathrm{E}-18$ & $3.14 \mathrm{E}-22$ \\
\hline $\mathrm{AHX71782.1}$ & skp outer membrane protein & Goat & $6.58 \mathrm{E}-30$ & $4.25 \mathrm{E}-19$ & $1.80 \mathrm{E}-18$ & $3.14 \mathrm{E}-22$ \\
\hline $\mathrm{AHX71783.1}$ & skp outer membrane protein & Duck & $6.58 \mathrm{E}-30$ & $4.25 \mathrm{E}-19$ & $1.80 \mathrm{E}-18$ & $3.14 \mathrm{E}-22$ \\
\hline $\mathrm{AHX71784.1}$ & skp outer membrane protein & Chicken & $6.58 \mathrm{E}-30$ & $4.25 \mathrm{E}-19$ & $1.80 \mathrm{E}-18$ & $3.14 \mathrm{E}-22$ \\
\hline AHX71785.1 & skp outer membrane protein & Turkey & $6.58 \mathrm{E}-30$ & $4.25 \mathrm{E}-19$ & $1.80 \mathrm{E}-18$ & 3.14E-22 \\
\hline $\mathrm{AHX71786.1}$ & skp outer membrane protein & Rabbit & $6.58 \mathrm{E}-30$ & $4.25 \mathrm{E}-19$ & $1.80 \mathrm{E}-18$ & 3.14E-22 \\
\hline
\end{tabular}


sequences, which indicates that it is more significant in comparison to others (Table 1).

\section{The analysis of Physico-chemical properties}

The physico-chemical properties are important determinants of protein function. Hence to detect any possible similarities/ differences in the proteins encoded by skp, ompW and vacj from strains of $P$. multocida infecting different hosts, the computational analysis of physico-chemical properties was done. The analysis of physico-chemical properties included negatively charged amino acids, positively charged amino acids, molecular weight, Theoretical pI, instability index and grand average of hydropathicity (GRAVY). All proteins listed in the (Table 2) play an important role in causing the infection in different animals with the help of carrier bacterial strains that is the host genera of Pasteurella multocida. The analysis showed that vacJ and ompW proteins in domesticated ruminant mammal sheep and goat are interestingly different in comparison to the proteins from strains of $P$. multocida infecting other animals, whereas, skp outer membrane protein is found to be similar in all the cases. The instability index of all proteins is less than 40 which means they are probably stable proteins but ompW encoded protein AHX71787.1_1 of sheep is probably more stable than others since the value is lesser. (13.12). The grand average of hydropathicity of the sequences calculates the sum of hydropath values among all protein sequences. Less score of GRAVY indicates less hydropathicity in a protein sequence. Increasing positive score of GRAVY shows greater hydropathicity. The protein encoded by skp gene shows smaller GRAVY score and thus less hydropathicity as compared to others. The major difference in the GRAVY between all other sequences with two protein sequences i.e.AHX71793.1_1Rabbit, AHX71787.1_1-Sheep encoded by ompW gene in the GRAVY value indicates greater hydropathicity of ompW encoded proteins (GRAVY value: 0.209). The differences between two protein sequences (AHW46103.1_1_sheep), (ahw46105.1_1_goat) encoded by vacj outer membrane protein family have comparatively less positive value of isoelectric point (6.62) which indicates less solubility of the encoded proteins (Table 2).

\section{Transmembrane helices}

The transmembrane helices affect the final three dimensional structure of the protein as Laos the signalling molecules that interact with the proteins. Thus with the aim of detecting similarities/ differences in the skp, vacJ and ompW proteins from strains of $P$. multocida infecting different hosts, the transmembrane helices (TMHs) including the number of amino acid present in TMHs were predicted. The number of TMHs is 0 (zero) in VacJ outer membrane lipoprotein family and 1 (one) in all protein sequences of ompW and skp outer membrane lipoprotein. The number of amino acids is approximately above 13 in group of vacJ outer membrane lipoprotein family and 17-19 amino acids are present in TMHs of ompW and skp outer membrane lipoprotein family. In protein (AHX71792.1_turkey) of ompW outer membrane protein family the number of predicted TMHs is 2 and number of amino acids in TMHs is approximately 41. In protein (AHX71790.1_duck) of ompW family the number of amino acid in the TMHs is 27.90096. The prediction confirms that all protein is outer membrane proteins (Table 3 ).

\section{Twin-arginine signal peptides}

It has been shown in other studies that the twin arginine signal peptides have the capability to identify the folded state of a substrate protein and to eliminate unfolded proteins. The substrates of TAT signal peptide pathway are frequently redox cofactor binding proteins which obtain the cofactor and therefore fold in cytoplasm. With the aim of detecting any similarities/differences in the signalling properties of vacJ, ompW and skp proteins from P. multocida strains infecting different hosts we predicted the twin arginine signal peptides in vacJ, ompW and SKP outer membrane proteins of Pasteurella multocida.

\begin{tabular}{|c|c|c|c|c|c|c|c|c|}
\hline ID & Protein Name & $\begin{array}{l}\text { Animal } \\
\text { name }\end{array}$ & $\begin{array}{c}\text { Number of negative } \\
\text { amino acids }\end{array}$ & $\begin{array}{c}\text { Number of positive } \\
\text { amino acids }\end{array}$ & $\begin{array}{l}\text { Molecular } \\
\text { weight }\end{array}$ & Theoretical pl & $\begin{array}{l}\text { Instability } \\
\text { Index }\end{array}$ & GRAVY \\
\hline AFQ32090.1_1 & vacJ outer membrane lipoprotein & Cattle & 28 & 29 & 27558.5 & 7.71 & 22.36 & -0.304 \\
\hline AFQ32091.1_1 & vacJ outer membrane lipoprotein & Duck & 28 & 29 & 27528.4 & 7.71 & 22.66 & -0.293 \\
\hline AFQ32092.1_1 & vacJ outer membrane lipoprotein & Chicken & 28 & 29 & 27528.4 & 7.71 & 22.66 & -0.293 \\
\hline AHW46101.1_1 & vacJ outer membrane lipoprotein & Quail & 28 & 29 & 27558.5 & 7.71 & 22.36 & -0.304 \\
\hline AHW46102.1_1 & vacJ outer membrane lipoprotein & Pig & 28 & 29 & 27528.4 & 7.71 & 22.66 & -0.293 \\
\hline AHW46103.1_1 & vacJ outer membrane lipoprotein & Sheep & 29 & 29 & 27612.5 & 6.62 & 24.27 & -0.311 \\
\hline AHW46104.1_1 & vacJ outer membrane lipoprotein & Buffalo & 28 & 29 & 27558.5 & 7.71 & 22.36 & -0.304 \\
\hline AHW46105.1_1 & vacJ outer membrane lipoprotein & Goat & 29 & 29 & 27612.5 & 6.62 & 24.27 & -0.311 \\
\hline AHW46106.1_1 & vacJ outer membrane lipoprotein & Rabbit & 28 & 29 & 27585.5 & 7.71 & 25.54 & -0.307 \\
\hline AHX71787.1_1 & Outer membrane protein $\mathrm{W}$ & Sheep & 15 & 19 & 21940.3 & 9.16 & 13.12 & 0.209 \\
\hline AHX71788.1_1 & outer membrane protein $\mathrm{W}$ & Buffalo & 14 & 19 & 21911.2 & 9.3 & 16.42 & 0.197 \\
\hline AHX71789.1_1 & outer membrane protein $\mathrm{W}$ & Goat & 14 & 19 & 21911.2 & 9.3 & 16.42 & 0.197 \\
\hline AHX71790.1_1 & outer membrane protein $\mathrm{W}$ & Duck & 13 & 19 & 21973.3 & 9.42 & 18.99 & 0.194 \\
\hline AHX71791.1_1 & outer membrane protein $\mathrm{W}$ & Chicken & 15 & 19 & 21912.2 & 9.16 & 13.53 & 0.197 \\
\hline AHX71792.1_1 & outer membrane protein $\mathrm{W}$ & Turkey & 13 & 19 & 21920.3 & 9.38 & 17.67 & 0.17 \\
\hline AHX71793.1_1 & outer membrane protein $\mathrm{W}$ & Rabbit & 15 & 19 & 21940.3 & 9.16 & 13.12 & 0.209 \\
\hline AHX71780.1_1 & skp outer membrane protein & Sheep & 31 & 33 & 21408.4 & 8.55 & 38.95 & -0.501 \\
\hline AHX71781.1_1 & skp outer membrane protein & Buffalo & 31 & 33 & 21408.4 & 8.55 & 38.95 & -0.501 \\
\hline AHX71782.1_1 & skp outer membrane protein & Goat & 31 & 33 & 21408.4 & 8.55 & 38.95 & -0.501 \\
\hline AHX71783.1_1 & skp outer membrane protein & Duck & 31 & 33 & 21408.4 & 8.55 & 38.95 & -0.501 \\
\hline AHX71784.1_1 & skp outer membrane protein & Chicken & 31 & 33 & 21408.4 & 8.55 & 38.95 & -0.501 \\
\hline AHX71785.1_1 & skp outer membrane protein & Turkey & 31 & 33 & 21408.4 & 8.55 & 38.95 & -0.501 \\
\hline AHX71786.1_1 & skp outer membrane protein & Rabbit & 31 & 33 & 21408.4 & 8.55 & 38.95 & -0.501 \\
\hline
\end{tabular}

Table 2: The analysis of Physico-chemical properties. 


\begin{tabular}{|c|c|c|c|c|}
\hline Protein_id & Protein Name & Animal name & Predicted TMH & Number of Amino Acid in the TMHs \\
\hline AFQ32090.1 & vacJ outer membrane lipoprotein & Cattle & 0 & 13.82271 \\
\hline AFQ32091.1 & vacJ outer membrane lipoprotein & Duck & 0 & 13.8215 \\
\hline AFQ32092.1 & vacJ outer membrane lipoprotein & Chicken & 0 & 13.8215 \\
\hline AHW46101.1 & vacJ outer membrane lipoprotein & Quail & 0 & 13.82271 \\
\hline AHW46102.1 & vacJ outer membrane lipoprotein & Pig & 0 & 13.8215 \\
\hline AHW46103.1 & vacJ outer membrane lipoprotein & Sheep & 0 & 13.62808 \\
\hline AHW46104.1 & vacJ outer membrane lipoprotein & Buffalo & 0 & 13.82271 \\
\hline AHW46105.1 & vacJ outer membrane lipoprotein & Goat & 0 & 13.62808 \\
\hline AHW46106.1 & vacJ outer membrane lipoprotein & Rabbit & 0 & 13.60224 \\
\hline AHX71787.1 & Outer membrane protein $\mathrm{W}$ & Sheep & 1 & 19.00975 \\
\hline AHX71788.1 & outer membrane protein $\mathrm{W}$ & Buffalo & 1 & 19.24359 \\
\hline AHX71789.1 & outer membrane protein $\mathrm{W}$ & Goat & 1 & 19.24359 \\
\hline AHX71790.1 & outer membrane protein $\mathrm{W}$ & Duck & 1 & 27.90096 \\
\hline AHX71791.1 & outer membrane protein $\mathrm{W}$ & Chicken & 1 & 18.96284 \\
\hline AHX71792.1 & outer membrane protein $\mathrm{W}$ & Turkey & 2 & 40.95429 \\
\hline AHX71793.1 & outer membrane protein $\mathrm{W}$ & Rabbit & 1 & 19.00975 \\
\hline AHX71780.1 & skp outer membrane protein & Sheep & 1 & 17.53972 \\
\hline AHX71781.1 & skp outer membrane protein & Buffalo & 1 & 17.53972 \\
\hline AHX71782.1 & skp outer membrane protein & Goat & 1 & 17.53972 \\
\hline AHX71783.1 & skp outer membrane protein & Duck & 1 & 17.53972 \\
\hline AHX71784.1 & skp outer membrane protein & Chicken & 1 & 17.53972 \\
\hline AHX71785.1 & skp outer membrane protein & Turkey & 1 & 17.53972 \\
\hline AHX71786.1 & skp outer membrane protein & Rabbit & 1 & 17.53972 \\
\hline
\end{tabular}

Table 3: The prediction of transmembrane helices.

\begin{tabular}{|c|c|c|c|}
\hline Protein_id & Protein Name & $\begin{array}{c}\text { Animal } \\
\text { name }\end{array}$ & $\begin{array}{c}\text { TAT signal } \\
\text { peptide }\end{array}$ \\
\hline AFQ32090.1 & vacJ outer membrane lipoprotein & Cattle & 0 \\
\hline AFQ32091.1 & vacJ outer membrane lipoprotein & Duck & 0 \\
\hline AFQ32092.1 & vacJ outer membrane lipoprotein & Chicken & 0 \\
\hline AHW46101.1 & vacJ outer membrane lipoprotein & Quail & 0 \\
\hline AHW46102.1 & vacJ outer membrane lipoprotein & Pig & 0 \\
\hline AHW46103.1 & vacJ outer membrane lipoprotein & Sheep & 0 \\
\hline AHW46104.1 & vacJ outer membrane lipoprotein & Buffalo & 0 \\
\hline AHW46105.1 & vacJ outer membrane lipoprotein & Goat & 0 \\
\hline AHW46106.1 & vacJ outer membrane lipoprotein & Rabbit & 0 \\
\hline AHX71787.1 & Outer membrane protein W & Sheep & 0 \\
\hline AHX71788.1 & outer membrane protein W & Buffalo & 0 \\
\hline AHX71789.1 & outer membrane protein W & Goat & 0 \\
\hline AHX71790.1 & outer membrane protein W & Duck & 0 \\
\hline AHX71791.1 & outer membrane protein W & Chicken & 0 \\
\hline AHX71792.1 & outer membrane protein W & Turkey & 0 \\
\hline AHX71793.1 & outer membrane protein W & Rabbit & 0 \\
\hline AHX71780.1 & skp outer membrane protein & Sheep & 1 \\
\hline AHX71781.1 & skp outer membrane protein & Buffalo & 1 \\
\hline AHX71782.1 & skp outer membrane protein & Goat & 1 \\
\hline AHX71783.1 & skp outer membrane protein & Duck & 1 \\
\hline AHX71784.1 & skp outer membrane protein & Chicken & 1 \\
\hline AHX71785.1 & skp outer membrane protein & Turkey & 1 \\
\hline AHX71786.1 & skp outer membrane protein & Rabbit & 1 \\
\hline & The & & \\
\hline
\end{tabular}

Table 4: The prediction of Twin-arginine signal peptides.

Analysis revealed that single twin arginine signal peptides are presents only in 7 sequences of SKP outer membrane proteins but absent in all protein sequences of vacJ and ompW outer membrane protein. The TAT signal peptides from twin arginine translocation pathway activate the well characterized sec export pathway (general secretion route) which has recently been discovered in bacteria [13] (Table 4).

\section{ß-barrel outer membrane proteins}

The protein vacJ, skp, ompW of Pasteurella multocida strains belong to a family of outer membrane proteins that are widespread in Gramnegative bacteria. With the aim of analysing functional properties of vacJ, skp and ompW proteins, the $ß$-barrel outer membrane proteins were predicted in these proteins. The analysis of $\beta$-barrel outer membrane proteins suggests that ompW class of proteins may be involved in the defence of bacteria against various forms of environmental stress. The same activity for vacJ and skp outer membrane protein is absent. The analysis is also confirmed by the crystal structure of E. coli OmpW proteins to 2.7-A resolution. The structure shows that ompW forms an 8 -stranded $\beta$-barrel with a long and narrow hydrophobic channel that contains a bound $n$-dodecyl- $N, N$-dimethylamine- $N$-oxide detergent molecule [11]. The data suggest that members of the ompW family of Pasteurella multocida strains might be involved in the transport of small hydrophobic molecules across the bacterial outer membrane (Table 5).

\section{Discussion}

This study suggests common mechanism of action of the analysed proteins namely vacJ, ompW and skp proteins from strains of $P$. multocida infecting different organisms. This analysis confirms that all the outer membrane proteins are infectious in nature. Domain analysis conforms common mechanism of action. Domain COG2853 surface lipoprotein plays a role in cell envelope biogenesis, pfam04333 is required for intracellular spreading and PRK15091worksas transportervia outer membrane protein (http://www.ncbi.nlm.nih.gov/Structure/cdd/ wrpsb.cgi).

The analysis of physico-chemical properties shows that the value of instability index is less than 40 in all protein sequences which implies that they are probably stable proteins [14]. The protein AHX71787.1_1 of sheep encoded by ompW outer membrane protein is probably a very stable protein because the instability index is very less in comparison all protein sequences. In the grand average of hydropathicity analysis the 
Citation: Pandey N, Grover M, Rai A (2016) Conservation of Properties of Outer Membranes Protein Across Host Genera of Pasteurella multocida Suggests Common Mechanism of Action. Mol Biol 5: 162. doi:10.4172/2168-9547.1000162

Page 5 of 5

\begin{tabular}{|l|c|c|c|}
\hline Protein_id & Protein Name & $\begin{array}{c}\text { Animal } \\
\text { name }\end{array}$ & $\begin{array}{c}\text { Total number of } \\
\text { B-barrel outer } \\
\text { membrane proteins } \\
\text { predicted }\end{array}$ \\
\hline AFQ32090.1 & vacJ outer membrane lipoprotein & Cattle & 0 \\
\hline AFQ32091.1 & vacJ outer membrane lipoprotein & Duck & 0 \\
\hline AFQ32092.1 & vacJ outer membrane lipoprotein & Chicken & 0 \\
\hline AHW46101.1 & vacJ outer membrane lipoprotein & Quail & 0 \\
\hline AHW46102.1 & vacJ outer membrane lipoprotein & Pig & 0 \\
\hline AHW46103.1 & vacJ outer membrane lipoprotein & Sheep & 0 \\
\hline AHW46104.1 & vacJ outer membrane lipoprotein & Buffalo & 0 \\
\hline AHW46105.1 & vacJ outer membrane lipoprotein & Goat & 0 \\
\hline AHW46106.1 & vacJ outer membrane lipoprotein & Rabbit & 0 \\
\hline AHX71787.1 & Outer membrane protein W & Sheep & 1 \\
\hline AHX71788.1 & outer membrane protein W & Buffalo & 1 \\
\hline AHX71789.1 & outer membrane protein W & Goat & 1 \\
\hline AHX71790.1 & outer membrane protein W & Duck & 1 \\
\hline AHX71791.1 & outer membrane protein W & Chicken & 1 \\
\hline AHX71792.1 & outer membrane protein W & Turkey & 1 \\
\hline AHX71793.1 & outer membrane protein W & Rabbit & 1 \\
\hline AHX71780.1 & skp outer membrane protein & Sheep & 0 \\
\hline AHX71781.1 & skp outer membrane protein & Buffalo & 0 \\
\hline AHX71782.1 & skp outer membrane protein & Goat & 0 \\
\hline AHX71783.1 & skp outer membrane protein & Duck & 0 \\
\hline AHX71784.1 & skp outer membrane protein & Chicken & 0 \\
\hline AHX71785.1 & skp outer membrane protein & Turkey & 0 \\
\hline AHX71786.1 & skp outer membrane protein & Rabbit & 0 \\
\hline & & & 0 \\
\hline
\end{tabular}

Table 5: The prediction of ß-barrel outer membrane proteins.

value of GRAVY is maximum in AHX71795.1_Rabbit, AHX71787.1_ Sheep encoded by ompW. The two protein sequences (AHW46103.1_1_ sheep), (ahw46105.1_1_goat) encoded by vacJ outer membrane protein family have comparatively less positive value of isoelectric point (6.62) which indicated that they may have less solubility . The analysis of transmembrane helices shows that the number of amino acids in the transmembrane helices is approximately above 13 in group of vacJ outer membrane lipoprotein family and 17-19 amino acids in ompW and skp outer membrane lipoprotein family. In protein (AHX71792.1_ turkey) of ompW outer membrane protein family the predicted TMHs is 2 and number of amino acid in TMHs is approximately 41.The prediction conforms that all protein are outer membrane proteins. The analysis shows that only skp membrane proteins show the presence of twin arginine signal peptides. B -barrels are present only in the ompW proteins.

\section{Conclusion}

P. multoicda infects a variety of animals and birds. The proteins vac J, omp W and skp proteins from P. multocida are important membrane proteins. In this study we asked whether these proteins have common mechanism of action in P. multocida strains infecting different host genera. Multiple sequence alignment, conserved domain analysis, analysis of physico-chemical properties, transmembrane helices, twin arginine signal peptides and $B$-barrel outer membrane proteins indicates that the mechanism of action of ompW, skp and vac J proteins is conserved across the P. multocida strains infecting different genera.
This information may be useful in developing a common vaccine for the diseases caused by P.multocida.

\section{References}

1. Boyce JD, Adler B (2000) The capsule is a virulence determinant in the pathogenesis of Pasteurella multocida M1404 (B:2). 2 Infect Immun 68: 34633468 .

2. Varga Z, Volokhov DV, Stipkovits L, Thuma A, Sellyei B, et al. (2013) Characterization of Pasteurella multocida strains isolated from geese. Vet Microbiol 163: 149-156

3. Ashraf A, Tariq H, Shah S, Nadeem S, Manzoor I, et al. (2011) Characterization of Pasteurella multocida strains isolated from cattle and buffaloes in Karachi, Pakistan. African Journal of Microbiology Research 5: 4673-4677.

4. Brambilla L, Morán-Barrio J, Viale AM (2014) Expression of the Escherichia coli ompW colicin $\mathrm{S} 4$ receptor gene is regulated by temperature and modulated by the H-NS and StpA nucleoid-associated proteins. FEMS Microbiol Lett 352 : 238-244.

5. Chaudhuri P, Singh V, Thamizharasan A, Lalsiamthara J (2012) Pasteurella multocida P52 aroA mutant conferred protection to rabbits and mice agains haemorrhagic septicaemia. DHR International Journal of Biomedical and Life Sciences 3, 127-136.

6. Chung JY, Wilkie I, Boyce JD, Townsend KM, Frost AJ, et al. (2001) Role of capsule in the pathogenesis of fowl cholera caused by Pasteurella multocida serogroup A. Infection and immunity 69: 2487-2492.

7. Shivachandra SB, Kumar A, Mohanty NN, Yogisharadhya R, Chacko N et al. (2014) Homogeneity of VacJ outer membrane lipoproteins among Pasteurella multocida strains and heterogeneity among members of Pasteurellaceae. Research in veterinary science $96: 415-421$

8. Holck A, Lossius I, Aasland R, Kleppe K (1987) Purification and characterization of the $17 \mathrm{~K}$ protein, a DNA-binding protein from Escherichia coli. Biochim Biophys Acta 914: 49-54

9. Suzuki T, Murai T, Fukuda I, Tobe T, Yoshikawa M, et al. (1994) Identification and characterization of a chromosomal virulence gene, vacJ, required for intercellular spreading of Shigella flexneri. Mol Microbiol 11: 31-41.

10. Volokhina EB, Grijpstra J, Stork M, Schilders I, Tommassen J, et al. (2011) Role of the periplasmic chaperones Skp, SurA, and DegQ in outer membrane protein biogenesis in Neisseria meningitidis. J Bacteriol 193: 1612-1621.

11. Ma J, Hart GW (2014) O-GlcNAc profiling: from proteins to proteomes. Clin Proteomics 11: 8 .

12. Neuberger G, Maurer-Stroh S, Eisenhaber B, Hartig A, Eisenhaber F (2003) Prediction of peroxisomal targeting signal 1 containing proteins from amino acid sequence. J Mol Biol 328: 581-592.

13. Ubarretxena-Belandia I, Engelman DM (2001) Helical membrane proteins: diversity of functions in the context of simple architecture. Current opinion in structural biology 11: 370-376.

14. Gasteiger E, Hoogland C, Gattiker A, Duvaud S, Wilkins MR, Appel RD, Bairoch A (2005) Protein Identification and Analysis Tools on the ExPASy Server. (In) John M. alker (ed): The Proteomics Protocols Handbook, Humana Press 571607 